\title{
THE PROPHYLACTIC APPROACH OF SESAMOL AND 3', 4'-(METHYLENEDIOXY)ACETOPHENONE TO PREVENT ASSOCIATED CARDIOTOXICITY OF DOXORUBICIN AT HIGH DOSE IN PROSTATE CANCER RAT MODEL
}

\author{
A. Shah ${ }^{1}$, A. A. Shah ${ }^{2}$, K. Nandakumar' ${ }^{2}$ D. S. Rekunge ${ }^{3}$, G. U. Chaturbhuj ${ }^{3}$, \\ A. Kishore ${ }^{2}$, P. G. Nayak ${ }^{2}$ and R. Lobo ${ }^{1 凶}$ \\ ${ }^{1}$ Department of Pharmacognosy, Manipal College of Pharmaceutical Sciences, Manipal \\ Academy of Higher Education, Manipal, 576104, Karnataka, India \\ ${ }^{2}$ Department of Pharmacology, Manipal College of Pharmaceutical Sciences, Manipal Academy \\ of Higher Education, Manipal, 576104, Karnataka, India \\ ${ }^{3}$ Department of Pharmaceutical Sciences and Technology, Institute of Chemical Technology, \\ Matunga, Mumbai, 400019, M.H., India \\ ${ }^{\square}$ Corresponding Author: richardlobo73@gmail.com
}

\begin{abstract}
The clinical application of Doxorubicin (DOX) is restricted due to the incidence of myelotoxicity and cardiotoxicity. Damages caused by free radicles to cardiac muscle tissues are more and permanent. The potent ROS scavenger, Sesamol (SM) has been proven to be effective in diseases related to oxidative stress. Thus, SM can be a good choice for myocardial protection against oxidative stress. 3',4'-(Methylenedioxy) acetophenone (3'MA) being derivative of SM have been proven in our lab to be antioxidant. Thus, both SM and 3'MA may be having ROS scavenging potential. In this study, we have examined the efficacy of SM and 3'MA protection against toxic effects of DOX at high dose administration in prostate cancer (PCa) induced rats. Results from the study indicate that doxorubicin $(4 \mathrm{mg} / \mathrm{kg})$ was potent to shows the anticancer activity in prostate cancer but also induce myocardial damage. The histopathological evaluation, Serum creatine kinase-muscle/brain (CK-MB) estimation, heart weight and tibia length ratio and haematology data suggest that the toxicity induced by DOX was prevented by SM and 3'MA treatment. Hence, the hypothesis proposed for this study and data generated from this study shows that SM and 3'MA can be drugs to augment the toxicity of DOX.

Keywords: Prostate Cancer, Doxorubicin, Cardiotoxicity, CK-MB, Sesamol, 3'MA
\end{abstract}

RASĀYAN J. Chem., Vol. 14, No.3, 2021

\section{INTRODUCTION}

Doxorubicin (DOX) is an anthracycline class antibiotic widely used for the treatment of various cancers including, breast, lung, leukaemia, lymphoma and other cancers. The clinical application of this drug is restricted due to the incidence of myelotoxicity and cardiotoxicity. There are several mechanisms reported in the literature to understand the various molecular pathways involved in DOX-induced cardiotoxicity. ${ }^{1}$ From the various studies, oxidative stress revealed the central role in the pathogenesis of myocardial damage and is closely associated with cardiac damage. ${ }^{2-4}$ Mitochondria are the main source for intracellular reactive oxygen species (ROS) generation such as hydroxyl $(\mathrm{OH}-)$ and superoxide $(\mathrm{O} 2 \bullet-)$ radicals, and hydrogen peroxide $(\mathrm{H} 2 \mathrm{O} 2) .{ }^{5}$ As cardiac tissue contains high concentrations of mitochondria; therefore, cardiac dysfunction arises due to increased mitochondrial oxidative stress. It is well known that myocardial tissues have very a much smaller number of antioxidants in comparison to other body tissues. Hence, damage caused by free radicles to cardiac muscle tissues are more and permanent. ${ }^{6,7}$

Since 1980, the dexrazoxane drug is approved for the treatment of DOX-induced cardiotoxicity. ${ }^{8,9}$ From 2017, the clinical use of dexrazoxane is contraindicated to children aged 0-18 years, and it may be associated with reversible toxicities, including myelosuppression. ${ }^{10}$ Therefore, there is increased attention in the research community towards alternative treatment options for DOX cardiotoxicity.

Rasayan J. Chem., 14(3), 1938-1946(2021)

http://doi.org/10.31788/RJC.2021.1436170

This work is licensed under a CC BY 4.0 license. 
Recently, naturally occurring antioxidants have gained attention to reduce the complications involved in oxidative stress. Several reports are indicating the effectiveness of antioxidant potential of natural compounds in DOX-induced cardiotoxicity. ${ }^{11-13}$

Despite the severe toxicity of DOX, it is continued in anticancer regimens. Many drugs have been investigated and are still investigating to prevent DOX toxicity and have synergistic potential as anticancer. Hence, we want to further explore Sesamol (SM) and 3',4'-(Methylenedioxy) acetophenone (3'MA) could inhibit cancer progression after the induction of prostate cancer (PCa). The gold standard drug treatment for $\mathrm{PCa}$ is docetaxel which is very expensive compared to DOX. As the study was selffunded we choose the alternative treatment drug DOX for our study. The hypothesis proposed was to evaluate the synergistic activity of SM and 3'MA along with DOX and also to evaluate the protective action of SM and 3' MA against DOX toxicity. The potent ROS scavenger, SM has been proven to be effective in diseases related to oxidative stress. On the other hand, SM as well plays a significant part in cancer by maintaining an oxidation-reduction balance. 3',4'-(Methylenedioxy) acetophenone (3'MA) being derivative of SM have been proven in our lab to be antioxidant. Thus, both SM and 3'MA may be having ROS scavenging potential. In this study, we have examined the efficacy of SM and 3'MA in PCa and protection against toxic effects of DOX administration in the rat. It has been hypothesized that improvement in oxidative stress by SM may be assisting in preventing the cardiotoxic effects of DOX and can be used for the management of DOX-associated complications.

\section{Materials and Methods}

EXPERIMENTAL

N-methyl N-nitrosourea (MNU) was synthesized by Mr. Deelip under the guidance of Dr. G. U. Chaturbhuj, ICT, Mumbai, Testosterone Undecanoate (TU) (Cernos Depot 1gm Injection) was purchased from Sun Pharmaceutical. Sesamol and 3',4'-(Methylenedioxy) acetophenone were purchased from Sigma-Aldrich. Rat PSA Prostate Specific Antigen (PSA) ELISA Kit (Catalog number: E-EL-R0796) is purchased from Elabscience. Doxorubicin hydrochloride (ADRIM, $25 \mathrm{ml}$ vial contains $50 \mathrm{mg}$ of DOX) is purchased locally. Serum CK-MB (Creatine Kinase-Muscle/Brain) kit (Catalog No.1102070210) is purchased from Coral clinical systems, Goa, India. All the analytical grade chemicals were procured from Merck Limited, India and Hi-media. Plastic labware and syringes were procured from tarson and BD biosciences respectively.

Animal grouping and experimental design for this purpose are given in Table-1.

Table-1: Animal Grouping and Treatment

\begin{tabular}{c|c}
\hline Experimental group & Treatment $(\mathrm{n}=6)$ \\
\hline Normal Control (NC) & Vehicle olive oil (i.p) $+0.25 \%$ CMC (p.o) \\
\hline Disease Control & MNU (50 mg/kg b.wt dissolved in PBS)+ Testosterone Undecanoate \\
(DC) & (TU) $(50 \mathrm{mg} / \mathrm{kg} ; 100 \mathrm{mg} / \mathrm{kg} ; 3 \mathrm{mg} / \mathrm{kg} ;$ i.p. $)$ in olive oil \\
\hline DOX Group & MNU+ TU + DOX $(5 \mathrm{mg} / \mathrm{kg})$ \\
\hline DOX+SM 100 & MNU+TU +DOX+ SM $(100 \mathrm{mg} / \mathrm{kg} \mathrm{p.o} \mathrm{in} 0.25 \%$ CMC) \\
\hline DOX+3' MA 100 & MNU+TU +DOX+3'MA $(100 \mathrm{mg} / \mathrm{kg}$ p.o in 0.25\% CMC) \\
\hline
\end{tabular}

Induction of Cardiotoxicity in PCa induced Rat's Preventive Effect of SM and 3' MA on it

The study was conducted in compliance with the committee for control and supervision of experiments on animals (CPCSEA) guidelines with the prior approval of the institutional animal ethical committee at the central animal research facility, Manipal) [Approval reference number - IAEC/KMC/27/2015]. Adult male Sprague Dawley rats (approximately 180-200 g) were used for conducting the experiments. PCa was induced by carcinogen using N-methyl N-nitrosourea (MNU) and Testosterone (TU) treatment for 16 weeks as described earlier ${ }^{14}$ DOX (4 mg/kg; i.p) was administered for the treatment of PCa and also associated with cardiotoxicity. As it is a preventive study, animals were treated with SM (100 mg $/ \mathrm{kg} \mathrm{p.o})$ and 3'MA (100 mg/kg p.o) suspended in carboxyl methylcellulose $(0.25 \%)$ after 16 weeks daily for 4 weeks, simultaneously with DOX $(4 \mathrm{mg} / \mathrm{kg})$ was administered for 4 weeks twice a week. The body weights of the animal were measured throughout the experiment. No animal died during experimentation, although, morbidity symptoms were seen in DOX control at the end stage of the study. Blood samples were collected by the retro-orbital method under mild anaesthesia. The serum was used to examine CK- 
MB cardiac markers with cardiac damage. Plasma was separated at $4000 \mathrm{rpm}\left(4{ }^{\circ} \mathrm{C}\right.$ for $\left.10 \mathrm{~min}\right)$ and stored at $-80{ }^{\circ} \mathrm{C}$ for further plasma parameters estimation. After $24 \mathrm{hr}$. of the last i. p. injection, rats were sacrificed, and prostate gland, heart as well as tibia was collected and weighed.

\section{Part I: Prostate Tumor-specific Markers \\ Prostate Weight: Prostate tissues were harvested, and weight was recorded. \\ Rat Plasma Prostate-Specific Antigen (PSA)estimation by ELISA Method}

The quantitative determination of Rat PSA concentrations in rat plasma based on Sandwich-ELISA principle. Precoated plates specific to Rat PSA were used. Sample/Standard added to the plate combined with the antibody. The plate is further treated with a biotinylated detection antibody and AvidinHorseradish Peroxidase (HRP) conjugate and incubated. The unbound components are washed away. The substrate solution is added to each well. Biotinylated detection antibody and Avidin-HRP conjugate, in the presence of rat PSA, will appear blue. Stop solution is added to terminate the enzyme-substrate reaction. It turns into a yellow color and is measured at $450 \mathrm{~nm} \pm 2 \mathrm{~nm}$ optical density (OD). The concentration of Rat PSA in the samples was calculated from the standard calibration curve.

\section{Rat Prostate Gland Histopathological Examination}

The tissue was fixed in 10\% formalin and $\mathrm{H}$ and $\mathrm{E}$ staining were performed by standard procedure.

\section{Part II: Cardiac Markers in Cardioprotective Study}

\section{Serum Creatine Kinase-muscle/Brain Estimation}

Enzymatic activities were measured according to the manufacturer's instructions using an auto-analyzer (Molecular Devices, Sunnyvale, CA, USA), and expressed as international units (U/1).

\section{Haematology Assessment}

Blood was collected from rats by retro-orbital route in eppendorf tubes containing $20 \mu 1$ of $10 \% \mathrm{w} / \mathrm{v}$ disodium EDTA as an anticoagulant. Tubes were gently turned upside down to mix the blood with anticoagulant. Blood parameters analyzed included haemoglobin ( $\mathrm{Hb})$, Mean cell volume (MCV), mean cell haemoglobin $(\mathrm{MCH})$ and Mean cell haemoglobin concentration (MCHC). These parameters were obtained using ERMA automated veterinary blood cell counter (model PCE-210 VET; Japan).

\section{Heart Weight: Tibia Length Ratio, Histopathology, and Antioxidant Assay}

Heart tissue was isolated and further dissected to isolate the left ventricle. Tissue weights and tibial lengths were measured using an analytical scale. One section of the heart was fixed in $10 \%$ formalin for histological examinations. Another section of the heart was kept in phosphate buffer saline, $\mathrm{pH} 7.4$ and stored at $-80{ }^{\circ} \mathrm{C}$ till further antioxidant analysis.

\section{Preparation of Heart Homogenate}

Heart tissue was homogenized in ice-cold PBS for homogenization at $2500 \mathrm{rpm}$ followed by centrifugation at $10,000 \mathrm{rpm}$ for $10 \mathrm{~min}$. After centrifugation, the supernatant was collected carefully without disturbing the sediment. The supernatant was collected and used for antioxidant assay estimations. The previously published procedure and standardized in our lab were used for the antioxidant estimations.

\section{Statistical Analysis}

The data were analyzed statistically and expressed as the mean $\pm \operatorname{SEM}(n=6 / 3)$. Groups were compared using ANOVA followed by Tukey's test for multiple comparisons. The level of significance is set at $\mathrm{p}<$ 0.05 .

Part I: Prostate Tumor-specific Markers

\section{RESULTS AND DISCUSSION}

\section{Prostate Weight}

Animals treated with TU and MNU (Disease control group) showed a significant increase in prostate weight of $79.61 \%$ (Fig.-1) compared to the normal control group. In the DOX-treated group, there is a 
RASĀYAN J. Chem.

Vol. 14 | No. 3 |1938-1946| July - September | 2021

drastic reduction in tumor weight with $47.23 \%$ as compared to the disease control group. It reveals the potent anticancer potential of DOX in the treatment of PCa. SM and 3'MA $(100 \mathrm{mg} / \mathrm{kg} / \mathrm{day}$, concomitantly administered with DOX after induction of PCa) decreased the prostate weight by $51.45 \%$ and $51.65 \%$. This weight reduction is comparable with DOX alone group (47.23\%). It indicates that SM and 3'MA have no significant influence on tumor weight reduction along with DOX treatment.

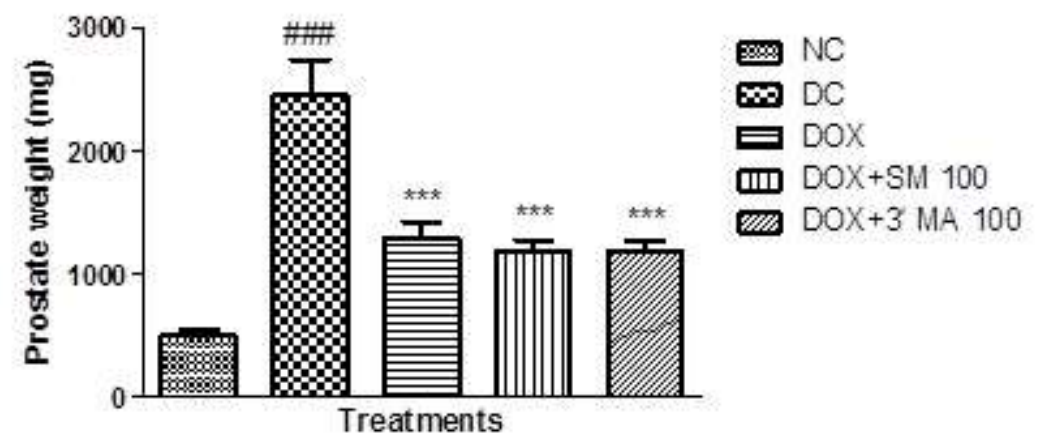

Fig.-1: Effect of SM and 3'MA concomitantly administered with DOX treatment on prostate weight \# represents a significant difference in means in comparison to normal control (NC) group data with the disease control (DC); while * indicates notable variation in means when compared to DC group with the test drug treatment groups.

\section{Rat Plasma Prostate-Specific Antigen (PSA) Estimation}

Prostate-specific antigen in serum is an early detection marker in PCa. PSA level is increased in the disease control group concerning normal control. It may be due to the diffusion of PSA in blood on the disruption of prostate epithelium. In the disease control group, there is $33.20 \%$ (Fig.-2) rise in PSA level as compared to the control group. While DOX treatment showed a reduction in PSA level $27.28 \%$ reduction. SM and 3'MA administered along with DOX did not show marked changes in PSA level as compared to DOX level. SM and 3'MA reduced PSA levels by $20.47 \& 22.53 \%$ respectively.

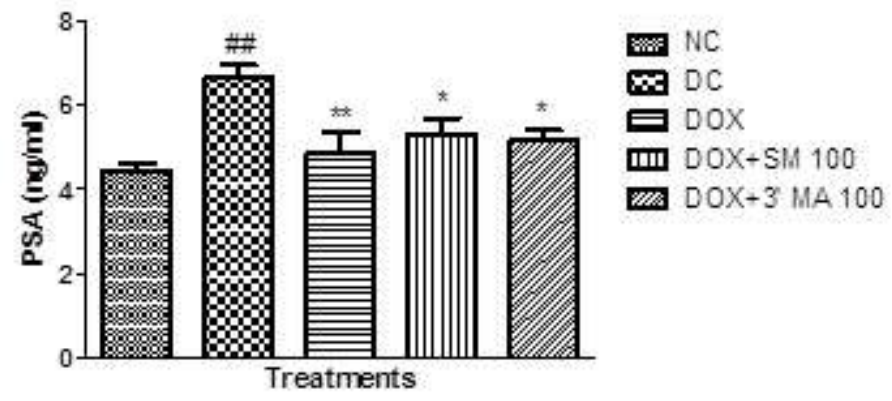

Fig.-2: Effect of SM and 3'MA concomitantly administered with DOX treatment on PSA level \# represents a significant difference in means in comparison to normal control (NC) group data with the disease control (DC); while * indicates notable variation in means when compared to DC group with the test drug treatment groups.

\section{Rat Prostate Gland Histopathological Examination}

Histopathological examination of prostate tissue confirmed the development of prostate cancer in the disease control group, whereas the DOX treated groups showed the recovery in tissue architect of the prostate(Fig.-3).

\section{Part II: Associated Myelotoxicity and Cardiac Toxicity Parameters Haematology Assessment}

Haematological parameters evaluation was performed on blood samples pretreated with EDTA. Results showed on DOX treatment there was a significant reduction in $\mathrm{WBC}, \mathrm{RBCs}, \mathrm{Hb}$, and platelets count and the following reduction in $\mathrm{MCV}, \mathrm{MCH}$, and MCHC (Table-2). 
RASĀYAN J. Chem.

Vol. 14 | No. 3 |1938-1946| July - September | 2021

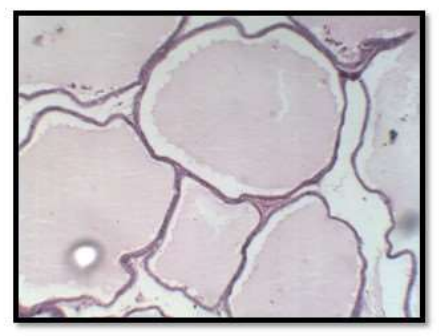

Normal Control

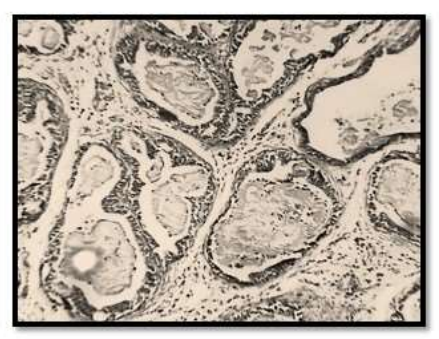

Disease Control

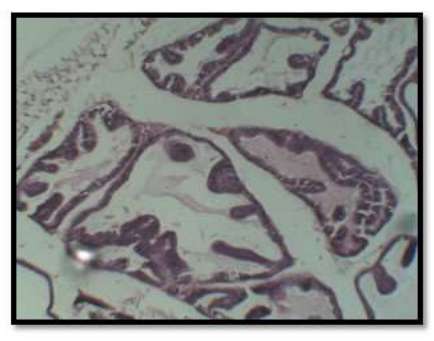

DOX

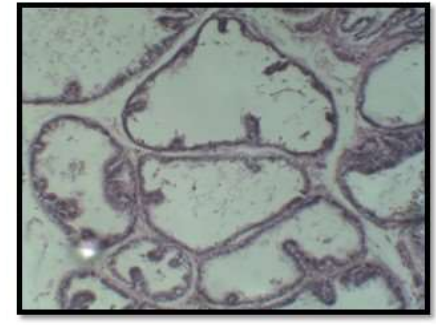

$\mathrm{DOX}+3$ ' MA 100

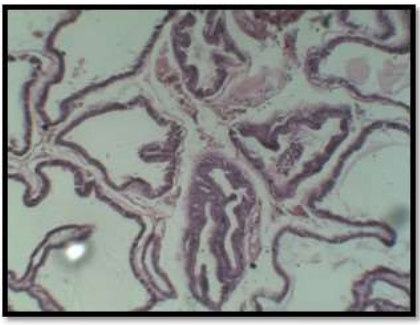

$\mathrm{DOX}+\mathrm{SM} 100$

Fig.-3: Histopathological Changes in prostate tissue following doxorubicin (DOX) treatment in SM-100 and 3'MA100 treated rat (100x magnification)

Table-2: Mean Hematological Parameters

\begin{tabular}{c|c|c|c|c}
\hline & $\mathrm{NC}$ & $\mathrm{DOX}$ & $\mathrm{DOX}+\mathrm{SM}$ & $\mathrm{DOX}+3^{\prime} \mathrm{MA} 100$ \\
\hline $\mathrm{WBC}\left(10^{3} / \mathrm{ul}\right)$ & $8.267 \pm 0.5812$ & $3.9 \pm 0.1528^{\# \# \#}$ & $6.467 \pm 0.4055^{* *}$ & $6.467 \pm 0.4177^{* *}$ \\
\hline $\mathrm{LY}\left(10^{3} / \mathrm{ul}\right)$ & $3.23 \pm 0.4702$ & $7.6 \pm 0.5859^{\# \# \#}$ & $3.1 \pm 0.1155^{* * *}$ & $3.4 \pm 0.3512^{* * *}$ \\
\hline $\mathrm{GR}\left(10^{3} / \mathrm{ul}\right)$ & $1.533 \pm 0.2848$ & $0.633 \pm 0.08819^{\#}$ & $1.533 \pm 0.1202^{*}$ & $1.767 \pm 0.1453^{* *}$ \\
\hline $\mathrm{RBC}\left(10^{6} / \mathrm{ul}\right)$ & $8 \pm 0.3007$ & $3.883 \pm 0.3023^{\# \# \#}$ & $7.42 \pm 0.1097^{* * *}$ & $7.36 \pm 0.1947^{* * *}$ \\
\hline $\mathrm{Hgb}(\mathrm{g} / \mathrm{dl})$ & $12.7 \pm 0.1453$ & $10.3 \pm 0.2517^{\# \#}$ & $11.9 \pm 0.0577^{* * *}$ & $11.8 \pm 0.1453^{* * *}$ \\
\hline $\mathrm{HCT}(\%)$ & $42.97 \pm 1.073$ & $26.17 \pm 1.26^{\# \#}$ & $42.53 \pm 0.4485^{* * *}$ & $41.4 \pm 0.8083^{* * *}$ \\
\hline $\mathrm{MCV}(\mathrm{fl})$ & $58.9 \pm 1.762$ & $49.73 \pm 0.2028^{\# \#}$ & $57.27 \pm 0.2603^{* *}$ & $56.2 \pm 0.4583^{* *}$ \\
\hline $\mathrm{MCH}(\mathrm{pg})$ & $14.7 \pm 0.1528$ & $10.43 \pm 0.3528^{\# \# \#}$ & $16 \pm 0.1528^{* * *}$ & $15.4 \pm 0.2517^{* * *}$ \\
\hline $\mathrm{MCHC}(\mathrm{g} / \mathrm{dl})$ & $29.6 \pm 0.4041$ & $22.2 \pm 0.2646^{\# \#}$ & $27.9 \pm 0.1667^{* * *}$ & $27.4 \pm 0.3786^{* * *}$ \\
\hline $\mathrm{PLT}\left(10^{3} / \mathrm{ul}\right)$ & $694 \pm 56.8$ & $373.3 \pm 20.38^{\# \#}$ & $637.3 \pm 42.23^{*}$ & $635 \pm 63.15^{*}$ \\
\hline
\end{tabular}

\# represents a significant difference in means in comparison to normal control (NC) group data with the disease control (DC); while * indicates notable variation in means when compared to DC group with the test drug treatment groups.

Anaemia along with impaired metabolism of iron are reported toxicity for DOX. Myocardial injury is linked with the cascade of inflammatory responses and observed with increased lymphocytes counts in the DOX-treated group which were replenished by SM and 3'MA treated group. The altered haematological parameters revealed the toxicity induced by doxorubicin. SM and 3'MA prevented the haematological parameters to get abnormal values explaining their myelo-protective action.

\section{SM and 3'MA Ameliorated Cardiac Injury}

$\mathrm{CK}-\mathrm{MB}$ is the predominant form of creatinine kinase expressed in heart tissue. CK-MB serum levels are increased in myocardial damage in several pathological conditions like ischemia, infarction. DOX treatment resulted in a dramatic increase in CK-MB levels. Treatment with SM and 3'MA along with DOX prevented a substantial increase in CK-MB levels (Fig.-4). 
RASĀYAN J. Chem.

Vol. 14 | No. 3 |1938-1946| July - September | 2021

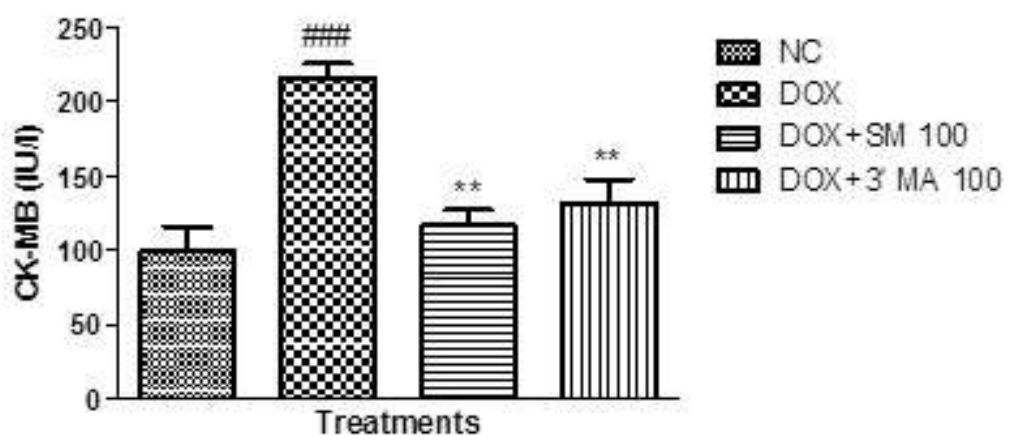

Fig.-4: Effect of SM and 3'MA on CK-MB level

\# represents a significant difference in means in comparison to normal control (NC) group data with the disease control (DC); while * indicates notable variation in means when compared to DC group with the test drug treatment groups.

\section{Heart Weight to Tibial Length (HW/TL) Ratio}

The heart-to-body weight ratio (HW/BW) was similar in all the groups. However, when comparing the heart weight to the tibial length (HW/TL) ratio, SM and 3'MA prevented HW/TL ratio, which was declined in the DOX-treated group (Fig.-5).

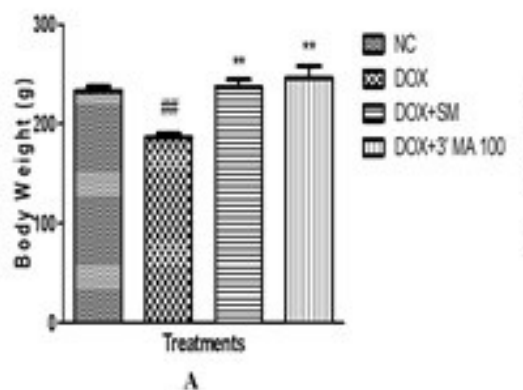

A
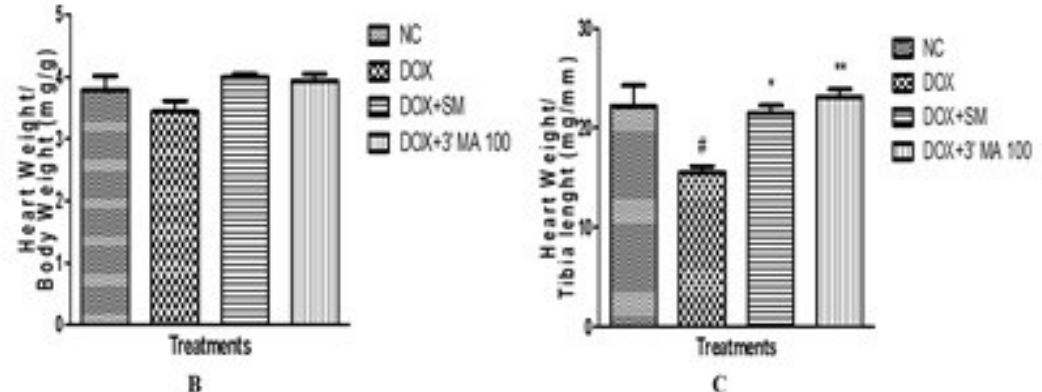

C

Fig.-5: Effect of SM and 3'MA on A) rat body weight B) Heart weight/Bodyweight ratio c) Heart weight to tibial length (HW/TL) ratio

\# represents a significant difference in means in comparison to normal control (NC) group data with the disease control (DC); while * indicates notable variation in means when compared to DC group with the test drug treatment groups.

\section{Effect of SM and 3'MA Treatment on Antioxidant Status of Heart Tissue}

Effect of SM and 3'MA treatment $(100 \mathrm{mg} / \mathrm{kg}$, orally, for 04 weeks) on-antioxidant markers as nitrite, lipid peroxidation, catalase, and GSH level were evaluated and quantified as mentioned in Table-3.

Table-3: Quantitative Estimation of Antioxidant Parameters in Heart Tissue Homogenate

\begin{tabular}{c|c|c|c|c}
\hline $\begin{array}{c}\text { Animal } \\
\text { Groups }\end{array}$ & $\begin{array}{c}\text { Nitrite (nmol of } \\
\text { CAT/mg protein) }\end{array}$ & $\begin{array}{c}\text { GSH (nmol of } \\
\text { GSH/mg protein }\end{array}$ & $\begin{array}{c}\text { LPO (nmol of } \\
\text { MDA /mg protein }\end{array}$ & $\begin{array}{c}\text { CAT (nmol of CAT/mg } \\
\text { protein) }\end{array}$ \\
\hline NC & $416 \pm 27.69$ & $0.4669 \pm 0.0978$ & $20.68 \pm 2.325$ & $0.5773 \pm 0.02862$ \\
\hline DOX & $1425 \pm 156.3^{\# \# \#}$ & $0.1197 \pm 0.0101^{\#}$ & $64.51 \pm 6.455^{\# \#}$ & $0.4565 \pm 0.03217$ \\
\hline $\begin{array}{c}\text { DOX+SM } \\
100\end{array}$ & $611.5 \pm 12.34 * * *$ & $0.73 \pm 0.1031^{* *}$ & $38.31 \pm 2.722^{* *}$ & $0.8554 \pm 0.05096^{* * *}$ \\
\hline $\begin{array}{c}\text { DOX+3' MA } \\
100\end{array}$ & $724.7 \pm 103.8^{* *}$ & $0.6866 \pm 0.07972^{* *}$ & $44.09 \pm 2.963^{*}$ & $0.6455 \pm 0.03707^{*}$ \\
\hline
\end{tabular}

DOX treatment resulted in an increase in lipid peroxidation as well as nitrite indicating damage to the antioxidant system in cardiac tissue of DOX treated rats. The glutathione and catalase provide a protective defence mechanism and impart their function against reactive oxygen species. The DOX induces cardiac 
RASĀYAN J. Chem.

Vol. 14 | No. 3 |1938-1946| July - September | 2021

oxidative stress by disturbing the oxidation-reduction balance. The SM and 3'MA proved to be cardioprotective by enhancing Catalase and glutathione (GSH) levels and reducing nitrite and MDA levels in cardiac tissue and thus maintaining oxidative stress.

\section{Heart Histopathological Examination}

The histopathological assessment was examined to estimate the pathological changes in cardiac tissue. The hematoxylin-eosin staining sections of DOX-treated animals showed marked toxicity visualized by morphological alterations in cardiac tissue. The muscle fibres distortions and degenerations, vacuolated muscle fibres, inflammatory infiltrations were signs of significant toxicity (Fig.-6).
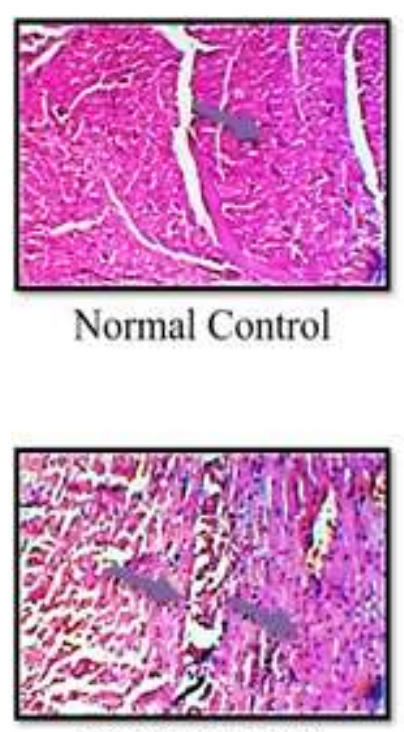

$\mathrm{DOX}+\mathrm{SM} 100$
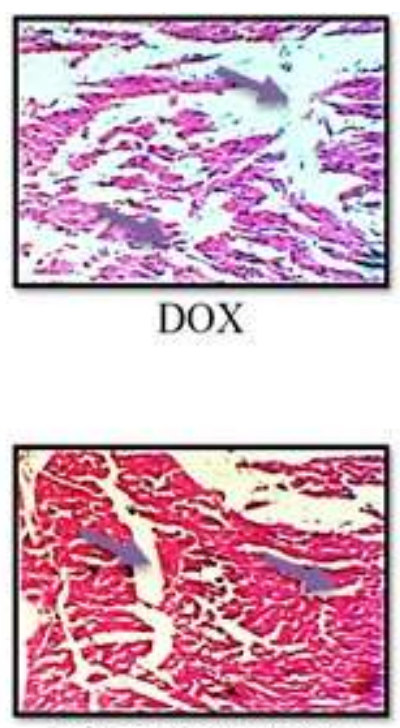

DOX+3'MA 100

Fig.-6: Histopathological changes in cardiac tissue following doxorubicin (DOX) treatment in SM-100 and 3'MA treated rat (100x magnification)

Cardiac tissue sections from SM and 3'MA (100 mg/kg b.w) with DOX group observed that myocardium was healthy in appearance, degeneration was less and no necrosis was found in comparison to sections of cardiac tissues taken from only DOX treated group.

\section{RESULTS AND DISCUSSION}

As per the available literature, the cardioprotective effect of sesamol $(50 \mathrm{mg} / \mathrm{kg} \mathrm{BW})$ is already reported in doxorubicin-induced cardiomyopathy in normal rats. Therefore, the present study investigated the possible cardioprotective role of it in PCa induced rats. DOX dose selected for the study was high to develop the cardiotoxicity and evaluate prevention of it by SM and 3' MA. DOX treatment in PCa animals showed a significant reduction in tumor weight and was found to be effective in PCa. But SM and 3'MA concomitantly administered along with DOX did not have a synergistic effect in reducing tumor weight. The histopathological estimation confirmed the development of $\mathrm{PCa}$ in the disease control group. Further, DOX treated groups regained the histoarchitecture of tissue to great extent. But there was no synergistic anticancer activity for DOX and SM or DOX and 3'MA.

PSA, the well-known glycoprotein is formed in the cells of the prostate, particularly in the prostatic epithelium but also by tumor cells. The serum levels of PSA is a parameter to access stages of prostate cancer and it is also used for the treatment strategies to determine the level of effects of treatments. ${ }^{14,15}$ Victor M. Garsky (2001) et. al; investigated the stabilizing role of Doxorubicin in prostate cancer; indicated by the reduced PSA level by $16 \% .{ }^{16}$ Similarly, the current investigating leads to decreased PSA level on DOX treatment. However, SM and 3'MA could not show a synergistic effect along with DOX 
RASĀYAN J. Chem.

Vol. 14 | No. 3 |1938-1946| July - September | 2021

treatments. Results from the previous studies indicated the preventive action of SM and 3'MA in PCa treatment option and can be used as an adjuvant therapy along with other chemotherapeutic drugs.

Therefore, the present study intended to study the effect of SM and 3'MA on myocardial damage in PCa rats for possible protective function. In PCa rats, histopathology of cardiac tissues resulted in observations like a myofibrillar loss as well as vacuoles formation in DOX treated rats, it is an indicator for cardiac toxicity.

SM is a potent antioxidant in nature. Based on the chemical nature of SM and being polyphenolic, it acts as an antioxidant and may be protected from the toxic effects of chemotherapy. Our results were also found to be reducing stress due to active free radicles of oxygen and potentiating antioxidant potential in the body. Thus, these facts claim SM to be a potent antioxidant and SM can be used as a drug to reduce the toxic effects of DOX like anticancer molecules. DOX caused the long-lasting focal inflammatory condition in the cardiac muscles (Fig.-5). SM-100 and 3'MA -treated rats showed recovery in the myocardial damage when compared to the DOX-treated cancer control group.

Reduced blood cell count is one of the primary indications for cardiac toxicity. DOX toxicity is revealed by the altered haematological parameters with a significant decrease in WBC, RBCs, Hb and platelets count. SM and 3'MA prevented the alteration of haematological parameters to the abnormal, indicate the protective function. Heart weight to tibia length ratio is one of the important markers in cardiac damage. Bodyweight is a poor reference parameter to use in assessing relative changes in heart weight in ageing or chronic hypertrophy like conditions. Tibia length is nearly independent of age changes and constant marker after maturity is used as an index in cardiac hypertrophy. ${ }^{17} \mathrm{SM}$ and 3'MA prevented heart weight to tibia length ratio declined by the DOX-treated group. Cardiac injury is also determined by serumrelated markers.

Creatinine kinase found in cytoplasm exists as a dimer. It has two subunits $\mathrm{M}$ and/or B. The isoenzymes that occur are CK-MM, CK-MB and CK-BB. Here, CK with suffix M and/or B is named as it occurs in tissues like $\mathrm{B}$ for the brain and $\mathrm{M}$ for skeletal muscles. The cardiac muscles have more CK-MM (60\%) while CK-MB is $40 \%$ and mitochondrial CK occurs in traces. They are highest in proportion in the myocardium and one of the important biochemical markers in patients with suspected acute myocardial infarction (AMI). Serum CK-MB is considered as the 'Gold Standard' for confirmation of the myocardial injury. Serum CK-MB level rises in the case of atrophy and myocardial infarction. ${ }^{18,19}$ In our investigation; there was an increased level of CK-MB in the DOX-treated group as compared with the normal control. SM and 3'MA influenced CK-MB levels and reduced in comparison with the DOXtreated group. ${ }^{20}$

Results from the study indicate that. SM and 3'MA (100 mg/kg p.o) were not effective in the treatment of PCa. Doxorubicin $(4 \mathrm{mg} / \mathrm{kg})$ was potent to shows the anticancer activity in prostate cancer but also induce myocardial damage. The histopathological evaluation, Serum creatine kinase-muscle/brain (CK-MB) estimation, heart weight and tibia length ratio and haematology data suggest that the toxicity induced by DOX was prevented by SM and 3'MA treatment. Therefore, SM and 3'MA decreased initial signs of cardiotoxicity, which may be mediated by suppressing oxidative stress.

\section{CONCLUSION}

The protective role of SM and 3'MA (100 mg/ $\mathrm{kg}$ ) was studied in the cardiotoxic model of DOX. The hypothesis proposed for this study and data generated from this study shows that SM and 3'MA can be drugs to augment the toxicity of DOX. The one proposed mechanism may be the antioxidant potential of SM and 3'MA. Although, the detailed mechanism needs to be studied for cardioprotective action.

\section{ACKNOWLEDGEMENT}

The authors' thanks to the Department of Pharmacognosy and Department of Pharmacology, Manipal College of Pharmaceutical Sciences and Manipal Academy of Higher Education, Manipal, for providing all the infrastructural facilities to carry out the research work.

\section{REFERENCES}

1. Y. Octavia, C.G. Tocchetti, K. L. Gabrielson, S. Janssens, H. J. Crijns, A. L. Moens, Journal of 1945 
RASĀYAN J. Chem.

Vol. 14 | No. 3 |1938-1946| July - September | 2021

molecular and cellular cardiology,52, 1213(2012), https://doi.org/10.1016/j.yjmcc.2012.03.006

2. M. F. Xu, P. L. Tang, Z. M. Qian, M. Ashraf. Life Science, 68, 889(2001), https://doi.org/10.1016/S0024-3205(00)00990-5

3. T. Simunek, M. Sterba, O. Popelova, M. Adamcova, R Hrdina, Pharmacological Reports 61, 154(2009), https://doi.org/10.1016/s1734-1140(09)70018-0

4. M. Songbo, H. Lang, C. Xinyong, X. Bin, Z. Ping, S. Liang. Toxicology Letter, 307, 41(2019), https://doi.org/10.1016/j.toxlet.2019.02.013

5. M. S. Tokarska, M. Zaugg, C. Zuppinger, T. Wallimann, U. Schlattner. Journal of molecular and cellular cardiology, 41, 389(2006), https://doi.org/10.1016/j.yjmcc.2006.06.009

6. J. Umlauf, M. Horký. Experimental \& Clinical Cardiology, 7, 35(2002)

7. Y. Ichikawa, M. Ghanefar, M. Bayeva, R. Wu, A. Khechaduri, S. V. N. Prasad, R. K. Mutharasan, T. J. Naik, H. Ardehali. Journal of Clinical Investigation, 124, 617(2014), https://doi.org/10.1172/JCI72931

8. D. Lebrecht, A. Geist, U-P. Ketelsen, J. Haberstroh, B. Setzer, U. A. Walker. British Journal of Pharmacology, 151, 771(2007), https://doi.org/10.1038/sj.bjp.0707294

9. P. Vejpongsa, E. T. H. Yeh. Journal of the American College of Cardiology, 64, 938(2014), https://doi.org/10.1016/j.jacc.2014.06.1167

10. P. Reichardt, M. D. Tabone, J. Mora, B. Morland, R. L. Jones. Future Oncology, 14, 2663(2018), https://doi.org/10.2217/fon-2018-0210

11. A. I. Abushouk, A. Ismail, A.M.A. Salem, A. M. Afifi, M. M. Abdel-Daim. Biomedicine Pharmacotherapy, 90, 935(2017), https://doi.org/10.1016/j.biopha.2017.04.033

12. A. Jain, V. Rani. 3 Biotech, 205, 97(2018), https://doi.org/10.1007/s13205-018-1425-6

13. J. Yu, C. Wang, Q. Kong, X. Wu, J. J. Lu, X. Chen. Phytomedicine 40, 125(2018), https://doi.org/10.1016/j.phymed.2018.01.009

14. G. Sharmila, T. Athirai, B. Kiruthiga, K. Senthilkumar, P. Elumalai, R. Arunkumar, J. Arunakaran. Nutrition and Cancer 66, 38(2014), https://doi.org/10.1080/01635581.2014.847967

15. M. Adhyam, A.K. Gupta. Indian Journal of Surgical Oncology, 3, 120(2012), https://doi.org/10.1007/s13193-012-0142-6

16. P. Srinivas, W Terry, P. Sahana, M. John, P John, P Sunjit. Biomedical Reports, 4, 263(2016), https://doi.org/10.3892/br.2016.586

17. V. M. Garsky, P. K. Lumma, D. M. Feng, J. Wai, H. G. Ramjit, M. K. Sardana, A. Oliff, R. E. Jones, D. DeFeo-Jones, R. M. Freidinger. Journal of Medicinal Chemistry, 44, 4216(2001), https://doi.org/10.1021/jm0101996

18. F. C. Yin, H. A. Spurgeon, K. Rakusan, M. L. Weisfeldt, E. G. Lakatta. American Journal of Physiology, 243, H941(1982), https://doi.org/10.1152/ajpheart.1982.243.6.H941

19. P. K. Nigam. Indian Journal of Clinical Biochemistry, 22, 10(2007). https://doi.org/10.1007/BF02912874

20. T. M. Welsh, G. D. Kukes, L.M. Sandweiss. Annals of Clinical \& Laboratory Science, 32, 44(2002)

[RJC-6170/2020] 